\title{
The suppression of hepatic glucose production improves metabolism and insulin sensitivity in subcutaneous adipose tissue in mice
}

\author{
Sylvie Casteras ${ }^{1,2,3}$ - Aya Abdul-Wahed ${ }^{1,2,3}$ - Maud Soty ${ }^{1,2,3}$ Fanny Vulin $^{1,2,3}$. \\ Hervé Guillou $^{4}$ • Mélanie Campana ${ }^{5,6}$ • Hervé Le Stunff ${ }^{5,6}$ • Luciano Pirola ${ }^{2,3,7}$. \\ Fabienne Rajas ${ }^{1,2,3}$ • Gilles Mithieux ${ }^{1,2,3}$ - Amandine Gautier-Stein 1,2,3
}

Received: 18 February 2016 / Accepted: 5 August 2016 /Published online: 9 September 2016

(C) Springer-Verlag Berlin Heidelberg 2016

\begin{abstract}
Aims/hypothesis Despite the strong correlation between nonalcoholic fatty liver disease and insulin resistance, hepatic steatosis is associated with greater whole-body insulin sensitivity in several models. We previously reported that the inhibition of hepatic glucose production (HGP) protects against the development of obesity and diabetes despite severe steatosis, thanks to the secretion of specific hepatokines such as fibroblast growth factor 21 (FGF21) and angiopoietinrelated growth factor. In this work, we focused on adipose tissue to assess whether liver metabolic fluxes might, by interorgan communication, control insulin signalling in lean animals.

Methods Insulin signalling was studied in the adipose tissue of mice lacking the catalytic subunit of glucose 6-phosphatase, the key enzyme in endogenous glucose production, in the
\end{abstract}

Electronic supplementary material The online version of this article (doi:10.1007/s00125-016-4097-y) contains peer-reviewed but unedited supplementary material, which is available to authorised users.

Amandine Gautier-Stein

amandine.gautier-stein@univ-lyon1.fr

1 Inserm U1213, Faculté Laennec, 7 rue Guillaume Paradin, 69372 Lyon cedex 08, France

2 Université de Lyon, Lyon, France

3 Université Lyon1, Villeurbanne, France

4 INRA, ToxAlim UMR1331 (Research Center in Food Toxicology), Toulouse, France

5 Unité Biologie Fonctionnelle et Adaptative -UMR CNRS 8251, Université Paris- Diderot (7), Paris, France

6 I2BC - UMR 9198 Université Paris Sud, Gif sur Yvette, France

7 Laboratoire de Recherche en Cardiovasculaire, Métabolisme, Diabétologie et Nutrition, CarMeN, Oullins, France liver (L-G6pc $c^{-/-}$mice). Morphological and metabolic changes in the adipose tissues were characterised by histological analyses, gene expression and protein content.

Results Mice lacking HGP exhibited improved insulin sensitivity of the phosphoinositide 3-kinase/Akt pathway in the subcutaneous adipose tissue associated with a browning of adipocytes. The suppression of HGP increased FGF21 levels in lean animals, and increased FGF21 was responsible for the metabolic changes in the subcutaneous adipose tissue but not for its greater insulin sensitivity. The latter might be linked to an increase in the ratio of monounsaturated to saturated fatty acids released by the liver.

Conclusions Our work provides evidence that HGP controls subcutaneous adipose tissue browning and insulin sensitivity through two pathways: the release of beneficial hepatokines and changes in hepatic fatty acids profile.

Keywords Adipose tissue $\cdot$ Fatty acids $\cdot$ Glucose 6-phosphatase $\cdot$ Hepatic glucose production $\cdot$ Hepatokines Insulin resistance $\cdot$ Liver $\cdot$ Obesity $\cdot$ Steatosis

$\begin{array}{ll}\text { Abbreviations } \\ \text { BAT } & \text { Brown adipose tissue } \\ \text { ChREBP } & \text { Carbohydrate-responsive element-binding } \\ & \text { protein } \\ \text { EpiWAT } & \text { Epididymal white adipose tissue } \\ \text { FGF21 } & \text { Fibroblast growth factor 21 } \\ \text { HGP } & \text { Hepatic glucose production } \\ \text { MUFA } & \text { Monounsaturated fatty acid } \\ \text { PI3K } & \text { Phosphoinositide 3-kinase } \\ \text { PPAR } \gamma & \text { Peroxisome proliferator-activated receptor } \\ & \text { gamma } \\ \text { SFA } & \text { Saturated fatty acid }\end{array}$




$\begin{array}{ll}\text { SWAT } & \text { Subcutaneous white adipose tissue } \\ \text { UCP1 } & \text { Uncoupling protein 1 } \\ \text { WT } & \text { Wild-type }\end{array}$

\section{Introduction}

Type 2 diabetes, which affects hundreds of millions of people worldwide, is characterised by a deregulation of hepatic glucose production (HGP), altered insulin secretion capacity and insulin resistance. Peripheral insulin resistance is mainly due to impaired insulin signalling in the muscles and adipose tissue [1]. Type 2 diabetes and more particularly insulin resistance are often diagnosed with related pathologies such as obesity and non-alcoholic fatty liver disease. The hepatic accumulation of triacylglycerol, which is a hallmark of nonalcoholic fatty liver disease, has been proposed as a causal factor leading to hepatic insulin resistance and to a further decrease in insulin sensitivity of the peripheral tissues. It is noteworthy that specific mechanisms, for example related to hepatokines, can dissociate hepatic steatosis from insulin resistance. For example, fetuin A (a hepatokine associated with steatosis and deleterious glucose control) is decreased in insulin-sensitive steatosis of liver-specific PTEN (phosphatase and tensin homolog) knockout mice [2]. Conversely, the increased production of fibroblast growth factor 21 (FGF21) is associated with insulin-sensitive steatosis [2-4]. Similarly, changes in lipid metabolism in the liver are sometimes associated with peripheral insulin sensitivity $[5,6]$. However, the mechanisms linking hepatic metabolism and the insulin-signalling pathway in peripheral tissues still need to be deciphered.

We recently showed that diverting the metabolic flux from gluconeogenesis into lipogenesis improves glucose control. Mice lacking the catalytic subunit of liver glucose 6-phosphatase (L-G6pc ${ }^{-/}$mice), the key enzyme of gluconeogenesis, exhibit hepatic steatosis associated with dramatic protection against diet-induced obesity and diabetes [7]. This protection is correlated with increased plasma FGF21 and angiopoietin-related growth factor concentrations [7]. To further document the relationship between HGP, FGF21 and insulin signalling, we focused on adipose tissue metabolism in L-G6pc ${ }^{-1-}$ mice. We report here that HGP suppression improved the insulin-signalling cascade in parallel to a white to brown conversion specifically in the subcutaneous white adipose tissue (SWAT). The increased secretion of FGF21 in L-G6pc ${ }^{-/-}$mice controlled the browning of the SWAT but surprisingly did not account for the enhanced insulin signalling. The latter seems to be related to an increased synthesis and release of monounsaturated fatty acids (MUFAs) in the liver of L-G6pc ${ }^{-/}$mice linked to an increase in carbohydrate-responsive element-binding protein (ChREBP) transcriptional activity.

\section{Methods}

Animals We used adult male L-G6pc $c^{-/-}$, I- $G 6 p c^{-/-}$, L-G6pc ${ }^{-1} . F g f 21^{-1-}$ [7-9] and wild-type (WT) (C57Bl/6J; Charles Rivers Laboratories, L'Arbresle, France) mice for the present studies. All mice were housed in the animal facility of Lyon 1 University (Animaleries Lyon Est Conventionnelle and Specific Pathogen Free) in controlled temperature $\left(22^{\circ} \mathrm{C}\right)$ conditions, with a $12 \mathrm{~h}$ light/ $12 \mathrm{~h}$ dark cycle. Mice had free access to water and standard rodent chow diet (A04; Safe, Augy, France). All procedures were performed in accordance with the principles and guidelines established by the European Convention for the Protection of Laboratory Animals. The regional animal care committee (CE2A-55, Lyon-1 University, France) approved all the experiments. Mice were killed in the middle of the dark period by cervical dislocation after injection of insulin $(0.38 \mathrm{U} / \mathrm{kg}$ body weight) or saline $(154 \mathrm{mmol} / \mathrm{l} \mathrm{NaCl})$.

Histological analyses Tissues were fixed and analysed as previously described [10]. Paraffin-embedded sections were incubated with anti-uncoupling protein 1 (UCP1) as previously described [10].

Immunoprecipitation assay and phosphoinositide 3-kinase activity Phosphoinositide 3-kinase (PI3K) activity associated with IRS1 or IRS2 (both from Santa Cruz Biotechnology, Heidelberg, Germany) was measured in lysates from tissues as previously described [11].

Western blotting Separation and blotting of $40 \mu \mathrm{g}$ samples of whole-tissue extracts were performed as previously described [12]. The source, characteristics and dilutions of the antibodies used are described in electronic supplementary material [ESM] Table 1.

Adipocyte culture Mice were killed in the middle of the dark period by cervical dislocation. Adipocytes were prepared from subcutaneous or epididymal fat pads as previously described [13]. Aliquots of $400 \mu \mathrm{l}$ of adipocytes cell suspension were incubated for $30 \mathrm{~min}$ with $100 \mathrm{nmol} / \mathrm{l}$ insulin (Sigma-Aldrich, Saint-Quentin Fallavier, France) and then for $30 \mathrm{~s}$ with 2 -[U- $\left.{ }^{14} \mathrm{C}\right]$ deoxy-D-glucose $(0.1 \mathrm{mmol} / 1,0.02 \mathrm{MBq} / \mathrm{ml}$-Perkin Elmer, Courtabeuf, France) as previously described [13]. Glucose uptake was expressed as dpm per $\mu \mathrm{g}$ of protein.

Gene expression analyses Total RNA was isolated from tissues with TRIzol reagent (Invitrogen, Cergy Pontoise, France). Reverse transcription and real-time PCR were performed as previously described [14]. The mouse ribosomal protein $\mathrm{mL} 19$ transcript (Rpl19) was used as a reference. The sequences of the specific primers used are available on request. 
FGF21 measurement Blood was withdrawn from the submandibular vein and collected in EDTA. FGF21 was assayed using ELISA kits from BioVendor (Abcys, Courtaboeuf, France).

Fatty acid composition Tissue lipid content and fatty acid composition were determined as previously described [15].

Measurement of ceramide levels Ceramide levels in the SWAT were measured using the diacylglycerol kinase enzymatic method as previously described [16].

Statistical analyses Results are reported as mean \pm SEM. Data were analysed with two-sample Student's $t$ tests or Kruskal-Wallis tests. Values were considered significant at $p<0.05$.

\section{Results}

Deletion of HGP improves insulin signalling in the subcutaneous adipose tissue To further document the relationship between HGP, FGF21 and insulin signalling, we studied insulin-signalling pathways in muscle, epididymal white adipose tissues (EpiWAT) and SWAT from L-G6pc $c^{-/}$and WT mice. We focused on the PI3K/Akt pathway, a key mediator of the effects of insulin on glucose metabolism.

L-G6pc $c^{-/-}$mice rapidly become hypoglycaemic under conditions of fasting [14] or insulin injection [7]. To avoid hypoglycaemia in L-G6pc $c^{-/-}$mice, we used low doses of insulin injected in the fed state $(0.38 \mathrm{U} / \mathrm{kg}$ body weight $)$. In these experimental conditions, insulin injection led to the same increase in PI3K activity and Akt phosphorylation levels in the EpiWAT (Fig. 1a, b) and gastrocnemius muscle (see [ESM] Fig. 1) of L-G6pc $c^{-/-}$mice as of WT mice. In parallel, insulin induced Akt phosphorylation only in the SWAT of L-G6pc $c^{-/-}$ mice, demonstrating enhanced insulin signalling in the SWAT of L-G6pc $c^{-/-}$compared with WT mice (Fig. 1d, e). In accordance with enhanced insulin signalling in the white adipose tissue of L-G6pc $c^{-/-}$mice, 2-deoxyglucose uptake by adipocytes from the L-G6pc $c^{-/-}$EpiWAT (Fig 1c) and SWAT (Fig 1f) was more strongly induced by insulin than was uptake by the WT adipocytes. The contents of the insulin receptor, the p85 regulatory subunit of the PI3K heterodimer and Akt were identical in the EpiWAT (Fig. 1g) and SWAT (Fig. 1h) of L-G6pc $c^{-1-}$ and WT mice.

In summary, the suppression of HGP promoted a higher activation of the PI3K/Akt pathway in response to insulin specifically in the SWAT, and an enhanced insulin-induced glucose uptake in the SWAT and EpiWAT. These results suggest the existence of crosstalk between the liver and the periphery, leading to modifications of insulin signalling.
Deletion of HPG induces modifications in adipose tissue metabolism Despite having a normal body weight [8], the L- $G 6 p c^{-1-}$ mice displayed a slight increase in SWAT mass (Fig. 2a) but no change in EpiWAT mass compared with WT mice (Fig. 2b). The levels of the peroxisome proliferator-activated receptor gamma (PPAR $\gamma$ ) and key PPAR $\gamma$ target genes - including genes implicated in adipogenesis (Cd36), glucose metabolism (Glut4 [also known as Slc2a4]) and adipokines (adiponectin, Adipoq) - were increased in the SWAT of L-G6pc $c^{-/-}$mice compared with WT mice (Fig. 2c, e) but unchanged in the EpiWAT of L-G6pc ${ }^{-/-}$ mice (Fig. 2d, f). Thus, suppression of HGP seems to mimic PPAR $\gamma$ activation, leading to changes in the expression of metabolic genes specifically in the SWAT.

Compared with WT SWAT, L-G6pc $c^{-1-}$ SWAT exhibited smaller, multilocular adipocytes, typical of brown adipose tissue (BAT) (Fig. 3a, b). Accordingly, UCP1 was induced in the SWAT of L-G6pc $c^{--}$mice (Fig. 3c). Although UCP1 levels were low in WT SWAT, UCP1 was preferentially produced in the small adipocyte population in L-G6pc $c^{-1-}$ mice (Fig. 3d, e). Finally, the expression of BAT-selective genes such as Cidea, Acoxl and Ucpl was increased twofold in the L-G6pc $c^{-1-}$ SWAT compared with WT mice (Fig. 3f). Thus, the small adipocyte population of the L-G6pc SWAT, which exhibited metabolic similarities to BAT, could be considered to be beige adipocytes. In agreement with the results on gene expression, EpiWAT morphology was not modified in L-G6pc $c^{-/-}$compared with WT mice (ESM Fig. 2).

Increase in FGF21 plasma levels induced by HGP suppression specifically controls browning in L-G6pc SWAT FGF21 is a liver-secreted factor that is involved in the control of energy metabolism. It has been linked to improved insulin sensitivity and to the regulation of several metabolic functions in adipose tissue. In adaptive thermogenesis, FGF21 regulates the browning of adipose tissue in an autocrine/paracrine manner [17]. We have previously demonstrated that suppression of HGP induced FGF21 production in the liver and FGF21 release in the plasma of L-G6pc ${ }^{-/}$mice fed a high-fat, high-sucrose diet [7]. The plasma concentration of FGF21, concomitantly with the content of FGF21 in the liver and SWAT, was increased in lean L-G6pc $c^{-/-}$compared with WT mice (Fig. 4a-c). In cultured adipocytes, FGF21 exerts a synergistic action with insulin on Akt phosphorylation, but no molecular mechanism has been described [18]. Interestingly, the overproduction of FGF21 decreases ceramide concentration, and ceramide, in turn, counteracts insulin signalling by the activation of protein phosphatase $2 \mathrm{~A}$, favouring Akt dephosphorylation [19]. Ceramide content was consistently decreased in the L-G6pc ${ }^{-/}$SWAT compared with the WT SWAT (Fig. 4d).

In light of the above results, we assessed whether knocking down Fgf21 [20] in L-G6pc $c^{-/-}$mice would abolish the 

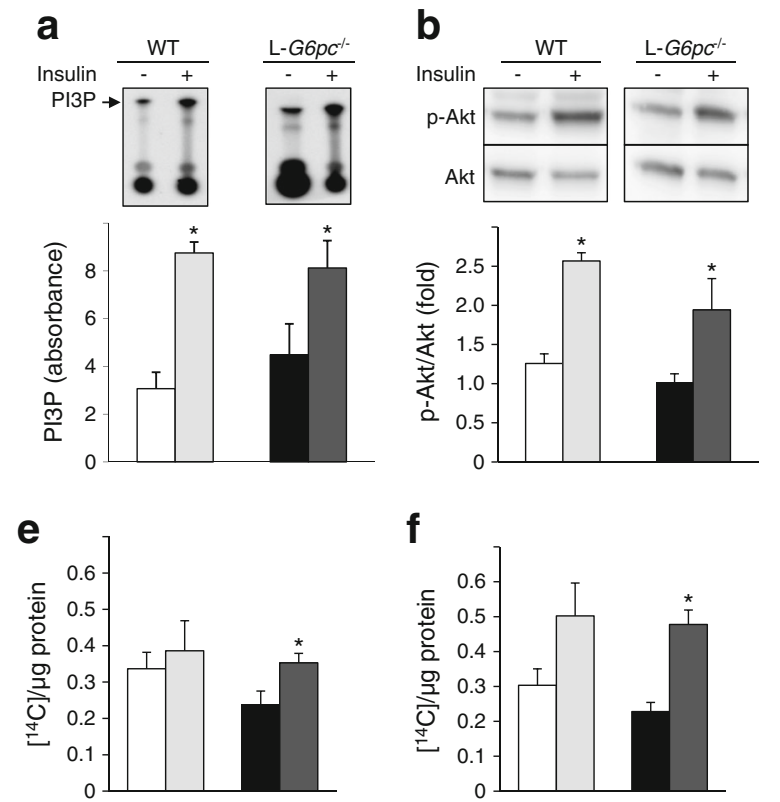

Fig. 1 PI3K/Akt signalling in the WAT of WT and L-G6pc ${ }^{-/}$mice. Quantification of IRS1-associated PI3K activity (i.e. amount of PI3P produced; absorbance, $\lambda=632.8 \mathrm{~nm}$ ) in the EpiWAT (a) and SWAT (c), and quantification of $\mathrm{pS}_{473}$-Akt/Akt protein in the EpiWAT (b) and SWAT (d) after insulin injection. Representative experiments are shown in each panel. Insulin-induced 2-deoxyglucose uptake by adipocytes from the WT and L- $G 6 p c^{-/-}$EpiWAT (e) and SWAT (f). Representative

changes in the metabolism and insulin signalling in the SWAT. L-G6pc ${ }^{-1-} . F g f 21^{-1-}$ double knockout mice did not express Fgf 21 (Fig. 4b, c). The SWAT of L-G6pc ${ }^{-/-} . F g f 21^{-/-}$mice did not exhibit the small adipocytes typical of BAT (Fig. 4e). The content of UCP1 and Pparg in the SWAT of L-G $6 p c^{-/-} . F g f 21^{-/-}$mice was restored to the level seen in WT mice and was much lower than that in L-G6pc ${ }^{-/-}$mice (Fig. 4f, g). In parallel, the ceramide content of the SWAT in L-G6pc ${ }^{-/-} . F g f 21^{-/-}$mice was also restored to the level seen in WT mice (Fig. 4d). However, the suppression of $F g f 21$ had no effect on the enhanced Akt phosphorylation induced by insulin in the L-G6pc ${ }^{-/-}$SWAT (Fig. 4h). In summary, Fgf 11 overexpression in L-G6pc ${ }^{-1-}$ mice was responsible for the morphological and metabolic changes but surprisingly not for the enhanced insulin signalling in the SWAT.

Increased intestinal gluconeogenesis induced by HGP suppression is not involved in the enhanced insulin signalling in SWAT We showed that suppression of HGP leads to the induction of intestinal gluconeogenesis [14]. The induction of gluconeogenesis improves whole-body insulin sensitivity through the production of a portal glucose signal, which targets hypothalamic nuclei [21]. Gluconeogenesis might thus be responsible for the enhanced insulin signalling in SWAT, a hypothesis that we tested using mice with a double knockout of G6pc in the

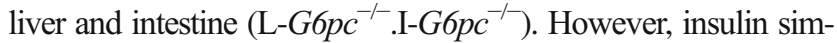
ilarly induced Akt phosphorylation in the SWAT of L-G6pc $c^{-/}$
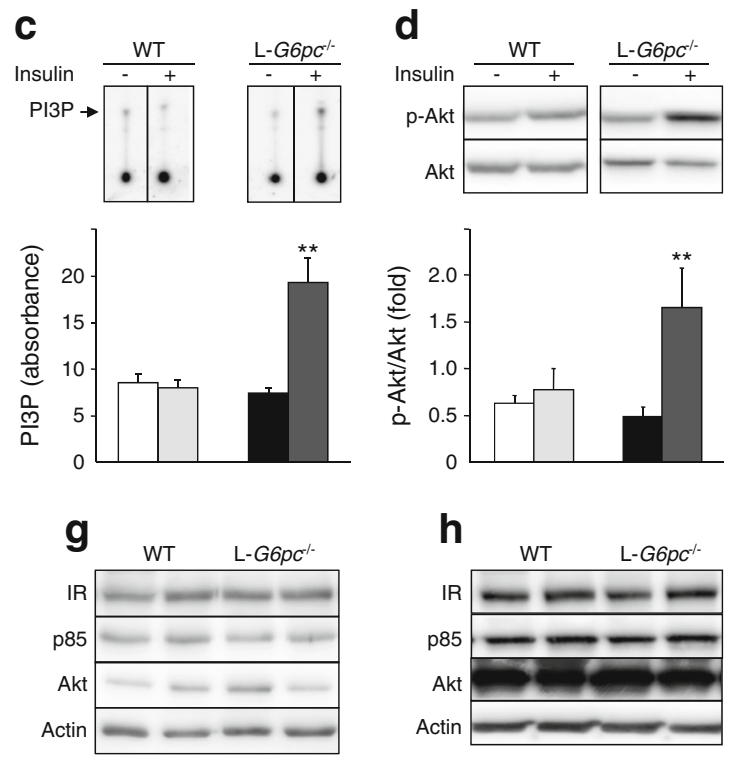

western blots showing the content of insulin receptor (IR), p85, Akt and actin in the EpiWAT (g) and SWAT (h) from WT and L-G6pc- $c^{--}$mice. White bars, WT mice; light grey bars, WT mice stimulated with insulin; black bars, L- $G 6 p c^{-/-}$mice; dark grey bars, L- $G 6 p c^{-1-}$ mice stimulated with insulin. PI3P, phosphatidylinositol 3-phosphate. Data are mean \pm SEM of five mice per group. Significant differences from the values obtained in non-insulin-injected mice, ${ }^{*} p<0.05$, $* * p<0.01$

mice (3.4 \pm 0.9 -fold induction by insulin) and in the SWAT of L-G $6 p c^{-/-}$.I-G $6 p c^{-/-}$mice (2.0 \pm 0.3 -fold induction by insulin).

Suppression of HGP increases the oleate/palmitate ratio in the liver We previously demonstrated that the specific inactivation of glucose 6-phosphatase in the liver leads to steatosis $[8,22]$, due to the transcriptional activation of ChREBP [7]. The suppression of HGP in lean mice also led to an increase in hepatic glucose 6-phosphate content (Fig. 5a) and Chrebp mRNA levels (Fig. 5b) with no changes in sterol regulatory element-binding protein (Srebp 1c) levels (Fig. 5c). In accordance with an induction of ChREBP transcriptional activity, the expression of key glycolytic and lipogenic genes was induced in the liver of lean L-G6pc ${ }^{-/}$compared with WT mice (Fig. 5c). Interestingly, the expression of $S c d 1$ and Elovl6, which are involved in the last step of de novo fatty acid synthesis and control the fatty acid composition of the liver, were also increased in the liver of L-G6pc $c^{-/-}$mice.

Fatty acid analysis revealed an increase in MUFA and a decrease in saturated fatty acid (SFA) content of the livers of L-G6pc ${ }^{-/-}$mice (Fig. 5d). More precisely, a specific increase in oleate $(\mathrm{C} 18: 1 \mathrm{n}-9)$ and a parallel decrease in palmitate $(\mathrm{C} 16: 0)$ were observed, leading to a higher ratio of oleate to palmitate in the liver of L-G6pc ${ }^{-1-}$ mice compared with WT mice (Fig. 5e). An increase in hepatic concentration of MUFAs (such as oleate) induced by the overproduction of ChREBP protects against SFA-mediated insulin resistance in primary hepatocytes 
a

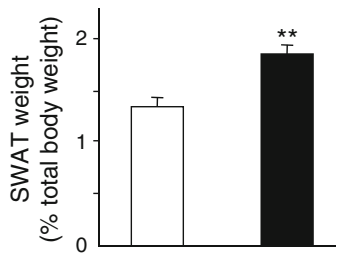

C
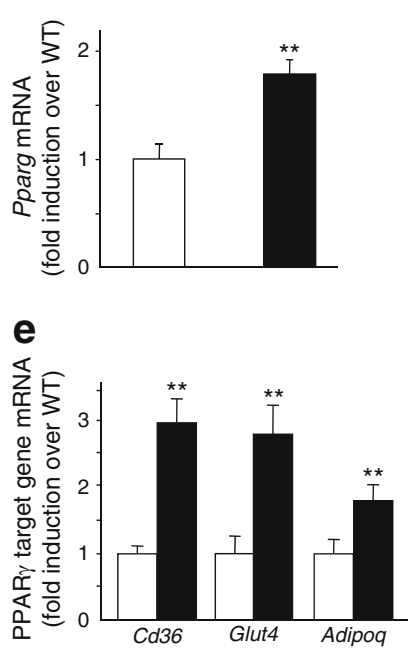

Fig. 2 Characteristics of white adipose tissue in L-G6pc $c^{-/-}$mice. Weight of the SWAT (a) and EpiWAT (b) expressed as a percentage of total body weight. Relative expression of Pparg mRNA in the SWAT (c) and EpiWAT (d), and PPAR $\gamma$ target genes in the SWAT (e) and EpiWAT (f). White bars, WT mice; black bars, L-G6pc ${ }^{-/-}$mice. Data are mean \pm SEM of eight mice per group. ${ }^{*} p<0.01$ vs WT values

[3]. As expected, the fatty acid composition of the plasma (Fig. 5g), the EpiWAT (Fig. 5h) and SWAT (Fig. 5i) mirrored the fatty acid composition of the liver, with a decrease in SFA and an increase in MUFA concentrations. High concentrations of circulating palmitate have deleterious effect on insulin signalling by inhibiting Akt phosphorylation in muscle and adipose tissue $[19,23]$. The increase in the oleate/palmitate ratio could thus account for the enhanced insulin signalling in the SWAT of L-G6pc $c^{-/-}$mice. In accordance with the latter hypothesis, the same induction of liver $S c d 1$ mRNA (Fig. 5f) and the same increase in the oleate/palmitate ratio were measured in the liver (Fig 5e), plasma (Fig. 5g) and WAT (Fig. 5h, i) of L-G6pc ${ }^{-/} . F g f 21^{-/-}$mice, which still exhibited an improved insulin signalling in the SWAT (Fig. 4h).

\section{Discussion}

Hepatic steatosis is widely accepted as a deregulation associated with obesity and type 2 diabetes. However, recent studies have demonstrated that triacylglycerol accumulation in the liver can be associated with improved insulin sensitivity, due to better glucose control in the peripheral tissues $[2,3,6,24$,
25]. To further document the mechanisms involved in this interorgan communication, we studied whether liver metabolism might control insulin signalling in the peripheral tissues. In this study, we focused on adipose tissue and report that the suppression of HGP, which is associated with severe steatosis, has beneficial effects on both insulin signalling and metabolism in the white adipose tissue by two independent mechanisms.

The suppression of HGP specifically induced the expansion and browning of the SWAT. Anatomically distinct adipose tissues differ substantially in their contributions to energy balance and nutrient homeostasis. The expansion of SWAT is associated with improved insulin sensitivity and physiological variables in obese mice [26] and can be protective against metabolic complications deriving from being fed a high-fat diet $[26,27]$. The metabolic benefit associated with SWAT expansion results from its capacity to contribute to thermogenesis through a white to brown conversion induced in response to cold exposure [28], $\beta 3$-adrenergic receptor agonist treatment [29], PPAR $\gamma$ agonist administration [30] or exposure to FGF21, irisin or cardiac natriuretic peptides [17, 31, 32]. Interestingly, de novo adipogenesis stimulated by cold exposure leads to different anatomical changes depending on the adipose tissue: a widespread browning of SWAT and a decrease in the EpiWAT mass [33], consistent with the results obtained in L-G6pc $c^{-/-}$mice. This differential effect might be due to a subset of transcription factors specifically produced in SWAT and controlling the expression of genes involved in the beige phenotype $[34,35]$.

We previously showed that the absence of HGP efficiently protects mice from the development of hyperglycaemia and obesity induced by a high-fat, high-sucrose diet, via the induction of adaptive thermogenesis in WAT, BAT and skeletal muscle [7]. These beneficial effects are mediated by interorgan communication induced by the release of two key hepatic regulators of peripheral metabolism: FGF21 and angiopoietin-related growth factor (AGF) [7]. In this study, the suppression of HGP in lean mice also increased FGF21 levels. In lean L-G6pc ${ }^{-/-}$mice, FGF21 induced a white to brown conversion in the SWAT, including an overproduction of UCP1 as a key endpoint. UCP1 levels were restored to normal in L-G6pc ${ }^{-/} . F g f 21^{-/-}$double knockout mice, which firmly indicates a causal role for FGF21 in the browning of SWAT in the L-G6pc $c^{-/-}$mice. Until recently, it was difficult to prove a role for beige adipocytes in controlling energy expenditure because of the supposedly dominant role played by BAT. However, recent studies highlight the potential importance of beige adipocytes in energy balance $[35,36]$. The decrease in HGP induced by the deletion of Pepck (also known as $P c k 1)$ specifically in the liver is also associated with hepatic steatosis and a compensatory increase in extrahepatic gluconeogenesis [37]. However, unlike in L-G6pc ${ }^{-/}$mice, the decrease in HGP in L-Pckl ${ }^{-/}$mice is associated with a parallel decrease in glucose utilisation in the peripheral tissues [37]. In L-Pckl ${ }^{-/}$ 
Fig. 3 Browning in the L-G6pc subcutaneous adipose tissue. Histological section of SWAT from WT (a, d) and L-G6pc $c^{-/}(\mathbf{b}, \mathbf{e})$ mice after HPS (haematoxylin phloxine saffron) staining (a, b) or UCP1 staining (d, e). Scale bars, $50 \mu \mathrm{m}$. (c) Representative western blots showing the content of UCP1 in the SWAT. (f) Relative mRNA expression of brown adipocyte markers in the SWAT; Pgcla is also known as Ppargcla. White bars, WT mice; black bars, L-G6pc $c^{-/}$ mice. Data are mean \pm SEM of six mice per group. ${ }^{* *} p<0.01$ vs WT values
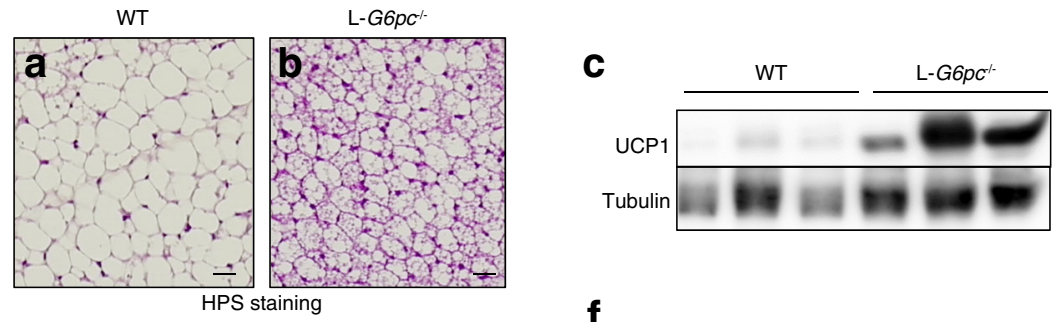

f

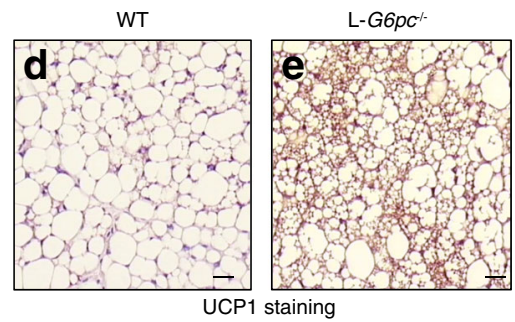

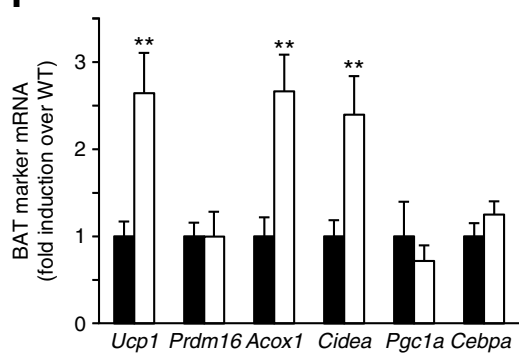

mice, the decrease in HGP does not induce an increase in either hepatic levels of glucose 6-phosphate or of its transcriptional target ChREBP or Fgf 21 expression [37]. This absence of increased plasma FGF21 levels may explain the opposite effects on peripheral tissue metabolism seen in the two models.
The main finding of this study is the demonstration that HGP controls insulin signalling in the SWAT. The induction of insulin signalling in the adipose tissue may play a quantitative role in the improvement of whole-body insulin sensitivity. Indeed, the specific deletion of PTEN in the adipose tissue induces Akt phosphorylation, leading to improved glucose
Fig. 4 FGF21 levels induced in L-G $6 p c^{-1-}$ mice. (a) FGF21 plasma levels. Relative mRNA levels of $F g f 21$ in the liver (b) and SWAT (c). (d) Ceramide content of the WT, L-G6pc ${ }^{-/-}$and L-G $6 p c^{-1-} . F g f 21^{-1-}$ SWAT. (e) Histological section of the WT, L-G $6 p c^{-/-}$and L-G6pc $c^{-/-}$. $F g f 21^{-/}$SWAT after HPS (haematoxylin phloxine saffron) staining. (f) Representative western blots showing the content of UCP1 in the SWAT. (g) Relative mRNA expression of Pparg in the SWAT. (h) Quantification of $\mathrm{pS}_{473}$-Akt over Akt protein measured in the SWAT. Representative western blots are shown on the top. White bars, WT mice; black bars, $\mathrm{L}-G 6 p c^{-/-}$mice; dark grey bars, L-G $6 p c^{-1-}$ mice stimulated with insulin; light grey bars, L-G6pc $c^{--} . F g f 21^{-1-}$ mice; striped bars, L-G6pc ${ }^{-1-} . F g f 21^{-1-}$ mice stimulated with insulin. Data are mean \pm SEM of five mice per group. $* * p<0.01$ vs WT mice, ${ }_{+} p<0.01$ vs L-G6pc $c^{-/-}$mice, ${ }^{\dagger} p<0.01$ vs non-insulin-injected mice
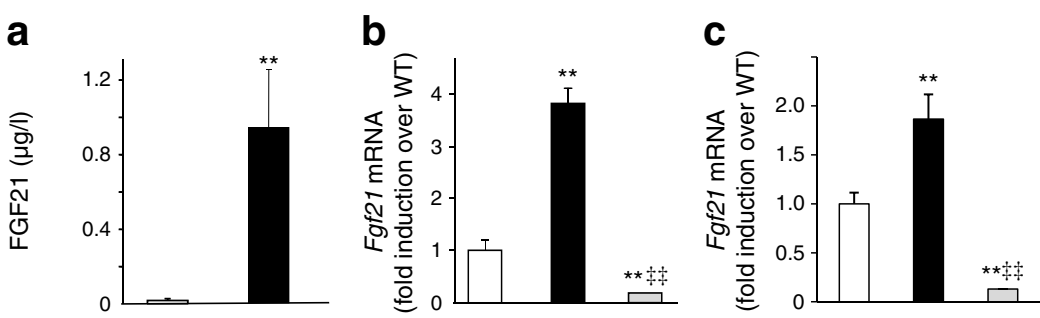

d

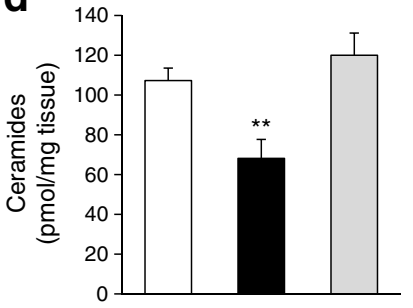

e

f
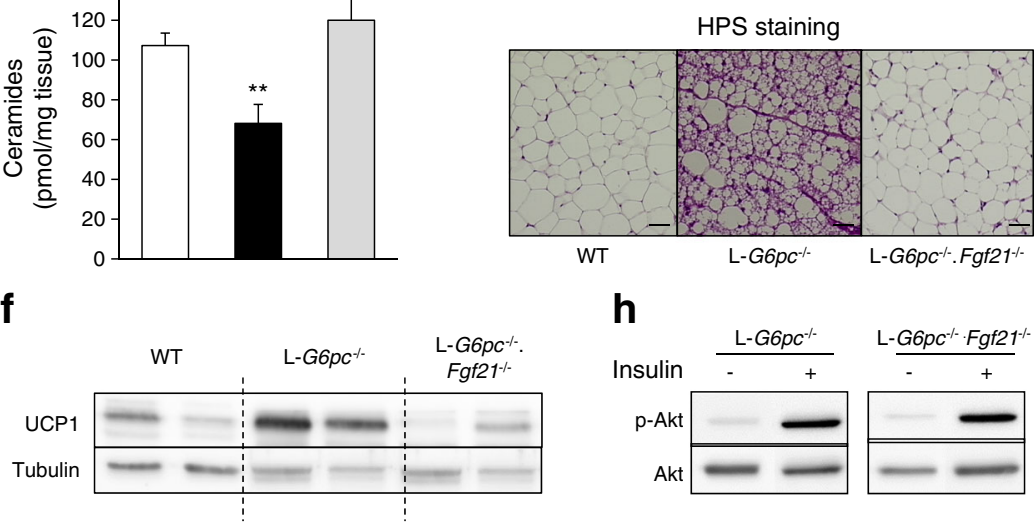

h

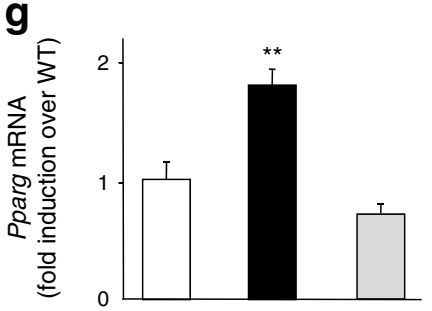


a

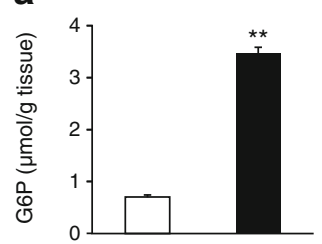

C
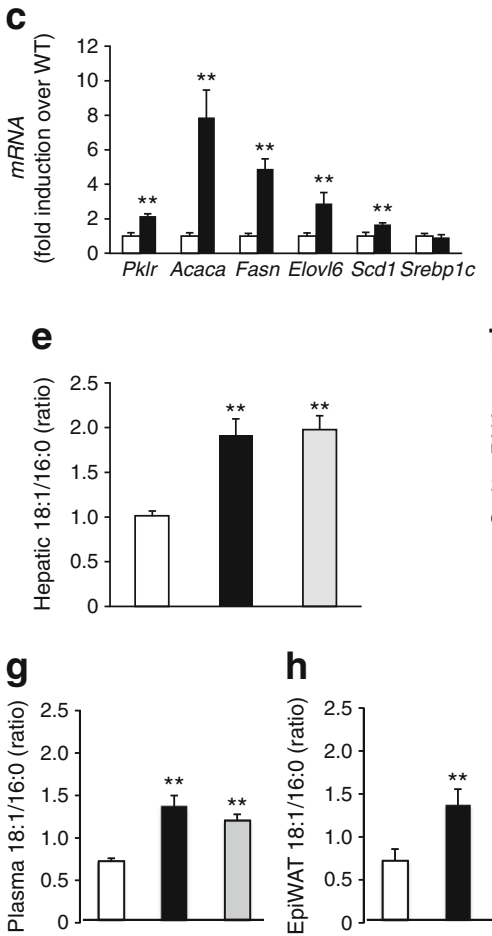

h
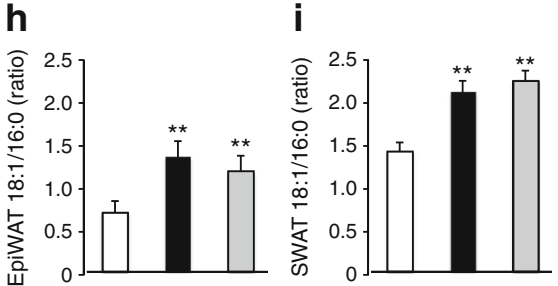

Fig. 5 Liver lipogenesis and fatty acids profile in L-G6pc $c^{-/-}$mice. (a) Hepatic glucose 6-phosphate (G6P) levels. (b) Relative Chrebp mRNA levels in the liver. (c) Relative mRNA levels of the genes of the glycolytic/lipogenic pathways in the liver. (d) SFA and MUFA levels in the liver, expressed as a percentage of the total liver fatty acids. (e) Hepatic C18:1/ $\mathrm{C} 16: 0$ ratio in WT, L-G6pc $c^{-/-}$and L-G6pc $c^{-/} . F g f 21^{-/}$mice. (f) Relative mRNA levels of $S c d 1$ in the liver. C18:1/C16:0 ratio in the plasma (g), EpiWAT (h) and SWAT (i) of WT, L-G6pc ${ }^{-/-}$and L-G6pc ${ }^{-/-} \cdot F g f 21^{-/-}$ mice. White bars, WT mice; black bars, L-G6pc $c^{-1-}$ mice; grey bars, $\mathrm{L}-G 6 p c^{-/-} . F g f 21^{-/-}$mice. Data are mean $\pm \mathrm{SEM}$ of five mice per group. $* * p<0.01$ vs WT

tolerance and insulin sensitivity [38]. Conversely, decreased Ser473 Akt phosphorylation specifically in the adipose tissue decreases GLUT4 export, disrupts glucose uptake in the adipose tissue and induces whole-body glucose intolerance [39]. GLUT4 is the major insulin-regulated glucose transporter and one of the main components of the insulin-signalling pathway [40]. In addition to its role in the regulation of glucose handling, GLUT4 expressed in the adipose tissue has recently been shown to be involved in the regulation of whole-body metabolism. The adipose tissue of mice overexpressing Glut4 produces a high level of fatty acyl hydroxy fatty acids, which are a novel class of endogenous lipids with anti-diabetic effects [41]. Thus, the increase in Glut4 expression and Akt phosphorylation in the L-G6pc ${ }^{-1-}$ SWAT might favour fatty acyl hydroxy fatty acid production. The increase in insulin signalling in the SWAT of L-Gopc $c^{-/-}$mice thus potentially goes beyond a better glucose uptake by the SWAT to induce the production of beneficial lipid species.

Several candidate factors may link the absence of HGP to insulin signalling in the SWAT. FGF21, secreted by liver, has been proposed as a regulator of insulin signalling in adipose tissue by acting in synergy with insulin on Akt phosphorylation [42]. However, the suppression of $F g f 21$ in L-G6pc $c^{-1}$ mice had no effect on insulin signalling in the SWAT, demonstrating that the metabolic changes induced by FGF21 do not control the insulin-signalling pathway. Intestinal gluconeogenesis improves whole-body insulin sensitivity and is induced in L-G6pc ${ }^{-1-}$ mice $[14,21]$. However, the additional suppression of gluconeogenesis in the latter had no effect on insulin signalling in the SWAT. An increased proportion of MUFAs in triacylglycerol is associated with a reduced risk of type 2 diabetes [43, 44]. Similarly, an increased ratio of MUFA to SFA in the liver is reflected in the plasma and is associated with insulin sensitivity even under conditions of extensive hepatic steatosis $[3,45,46]$. While the SFA palmitate alters the insulin signalling in the heart, adipose tissue and muscle (for a review, see [47]), the MUFA oleate exhibit protective effects against palmitate toxicity including an increase in insulin-induced Akt phosphorylation [48]. The beneficial effects of oleate have always been demonstrated in the context of specific diets. However, our study suggests that changes in liver metabolism might promote the same beneficial effects. Indeed, the suppression of HGP oriented the metabolic flux of glucose 6-phosphate toward the glycogenic and lipogenic pathways, which led to an increased MUFA/SFA ratio. In addition to the production of beneficial hepatokines, HGP suppression alters fatty acid metabolism in the liver, which could be responsible for the enhanced insulin-signalling pathway in the peripheral tissues. Interestingly, the control of white adipose tissue metabolism, particularly lipolysis, is also a key regulator of insulin sensitivity in the liver. Indeed, WATinduced lipolysis controls the suppression of HGP by insulin through the regulation of hepatic acetyl-CoA levels [49]. The bidirectional dialogue between the liver and WAT metabolism could thus be a key element in the regulation of whole-body insulin sensitivity.

In conclusion, our results suggest that the reorientation of glucose 6-phosphate flux toward the glycolytic/lipogenic pathway may have beneficial effects on peripheral tissues through two distinct mechanisms regulating energy expenditure via FGF21 and insulin signalling via the MUFA/SFA ratio. It is of note that all mouse models exhibiting a dissociation between hepatic steatosis and insulin resistance are characterised by an increase in hepatic FGF21 levels and MUFA/SFA ratio, and are never associated with an increase in HGP $[2,3,6]$. The latter observation reinforces the key role of increased HGP in the appearance of fasting hyperglycaemia 
associated with type 2 diabetes [7,50]. Our data thus highlight that the control of HGP, beyond hyperglycaemia, could be crucial in the management of the metabolic syndrome by the induction of energy expenditure and insulin signalling in the periphery.

Acknowledgements We thank the members of ALECS (Animalerie Lyon Est Conventionnelle et SPF, Faculté de Médecine Laennec, Structure Fédératrice de Recherche Lyon-Est, Lyon, France) for animal care, the members of the Anipath Platform (Universite Lyon1 Laennec, Lyon, France), D. Mangelsdorf and S. Kliewer (University of Texas Southwestern Medical Center, Dallas, TX, USA) for generously providing B6.Fgf $21^{-/-}$, and E Hajduch (Inserm UMRS 872, Paris, France) for help in primary culture of the adipose tissues.

Funding This work was supported by research grants from the Fondation pour la recherche médicale (grant No. DRM20121220448) and IFR62 Lyon-Est.

Duality of interest The authors declare that there is no duality of interest associated with this manuscript.

Contribution statement SC conducted and designed experiments, performed data analyses and wrote the manuscript, AAW, MS, MC, HLS, $\mathrm{HG}$ and FV acquired and analysed data and revised the manuscript, HLS, HG and LP contributed to the discussion and interpretation of data and revised the manuscript, GM, FR and AG-S contributed to the discussion and interpretation of data, supervised and edited the manuscript. All authors read and approved the final manuscript. AG-S is the guarantor of this work.

\section{References}

1. Samuel VT, Shulman GI (2012) Mechanisms for insulin resistance: common threads and missing links. Cell 148:852-871

2. Peyrou M, Bourgoin L, Poher A-L et al (2015) Hepatic PTEN deficiency improves muscle insulin sensitivity and decreases adiposity in mice. J Hepatol 62:421-429

3. Benhamed F, Denechaud PD, Lemoine M et al (2012) The lipogenic transcription factor ChREBP dissociates hepatic steatosis from insulin resistance in mice and humans. J Clin Invest 122: 2176-2194

4. Stefan N, Häring H-U (2013) The role of hepatokines in metabolism. Nat Rev Endocrinol 9:144-152

5. Monetti M, Levin MC, Watt MJ et al (2007) Dissociation of hepatic steatosis and insulin resistance in mice overexpressing DGAT in the liver. Cell Metab 6:69-78

6. Sun Z, Miller RA, Patel RT et al (2012) Hepatic Hdac3 promotes gluconeogenesis by repressing lipid synthesis and sequestration. Nat Med 18:934-942

7. Abdul-Wahed A, Gautier-Stein A, Casteras S et al (2014) A link between hepatic glucose production and peripheral energy metabolism via hepatokines. Mol Metab 3:531-543

8. Mutel E, Abdul-Wahed A, Ramamonjisoa N et al (2011) Targeted deletion of liver glucose- 6 phosphatase mimics glycogen storage disease type 1a including development of multiple adenomas. J Hepatol 54:529-537
9. Penhoat A, Mutel E, Amigo-Correig M et al (2011) Protein-induced satiety is abolished in the absence of intestinal gluconeogenesis. Physiol Behav 105:89-93

10. Rajas F, Jourdan-Pineau H, Stefanutti A et al (2007) Immunocytochemical localization of glucose 6-phosphatase and cytosolic phosphoenolpyruvate carboxykinase in gluconeogenic tissues reveals unsuspected metabolic zonation. Histochem Cell Biol 127:555-565

11. Fröjdö S, Cozzone D, Vidal H, Pirola L (2007) Resveratrol is a class IA phosphoinositide 3-kinase inhibitor. Biochem J 406:511-518

12. Gautier-Stein A, Domon-Dell C, Calon A et al (2003) Differential regulation of the glucose-6-phosphatase TATA box by intestinespecific homeodomain proteins CDX1 and CDX2. Nucleic Acids Res 31:5238-5246

13. Blouin CM, Prado C, Takane KK et al (2010) Plasma membrane subdomain compartmentalization contributes to distinct mechanisms of ceramide action on insulin signaling. Diabetes 59:600-610

14. Mutel E, Gautier-Stein A, Abdul-Wahed A et al (2011) Control of blood glucose in the absence of hepatic glucose production during prolonged fasting in mice: induction of renal and intestinal gluconeogenesis by glucagon. Diabetes 60:3121-3131

15. Zadravec D, Brolinson A, Fisher RM et al (2010) Ablation of the very-long-chain fatty acid elongase ELOVL3 in mice leads to constrained lipid storage and resistance to diet-induced obesity. Faseb J 24:4366-4377

16. Picard A, Rouch C, Kassis N et al (2013) Hippocampal lipoprotein lipase regulates energy balance in rodents. Mol Metab 3:167-176

17. Fisher FM, Kleiner S, Douris N et al (2012) FGF21 regulates PGC1 alpha and browning of white adipose tissues in adaptive thermogenesis. Genes Dev 26:271-281

18. Dutchak PA, Katafuchi T, Bookout AL et al (2012) Fibroblast growth factor-21 regulates PPARgamma activity and the antidiabetic actions of thiazolidinediones. Cell 148:556-567

19. Chavez JA, Summers SA (2003) Characterizing the effects of saturated fatty acids on insulin signaling and ceramide and diacylglycerol accumulation in 3T3-L1 adipocytes and $\mathrm{C} 2 \mathrm{C} 12$ myotubes. Arch Biochem Biophys 419:101-109

20. Potthoff MJ, Inagaki T, Satapati S et al (2009) FGF21 induces PGC1alpha and regulates carbohydrate and fatty acid metabolism during the adaptive starvation response. Proc Natl Acad Sci U S A 106: 10853-10858

21. Mithieux G, Andreelli F, Magnan C (2009) Intestinal gluconeogenesis: key signal of central control of energy and glucose homeostasis. Curr Opin Clin Nutr Metab Care 12:419-423

22. Ramamonjisoa N, Ratiney H, Mutel E et al (2013) In vivo hepatic lipid quantification using MRS at 7 Tesla in a mouse model of glycogen storage disease type 1a. J Lipid Res 54:2010-2122

23. Guo W, Wong S, Xie W et al (2007) Palmitate modulates intracellular signaling, induces endoplasmic reticulum stress, and causes apoptosis in mouse 3T3-L1 and rat primary preadipocytes. Am J Physiol Endocrinol Metab 293:E576-E586

24. Stiles B, Wang Y, Stahl A et al (2004) Liver-specific deletion of negative regulator Pten results in fatty liver and insulin hypersensitivity [corrected]. Proc Natl Acad Sci U S A 101:2082-2087

25. Uno K, Katagiri H, Yamada T et al (2006) Neuronal pathway from the liver modulates energy expenditure and systemic insulin sensitivity. Science 312:1656-1659

26. Kim JY, van de Wall E, Laplante M et al (2007) Obesity-associated improvements in metabolic profile through expansion of adipose tissue. J Clin Invest 117:2621-2637

27. Kusminski CM, Holland WL, Sun K et al (2012) MitoNEET-driven alterations in adipocyte mitochondrial activity reveal a crucial adaptive process that preserves insulin sensitivity in obesity. Nat Med 18:1539-1549 
28. Cousin B, Cinti S, Morroni M et al (1992) Occurrence of brown adipocytes in rat white adipose tissue: molecular and morphological characterization. J Cell Sci 103(Pt 4):931-942

29. Cao L, Choi EY, Liu X et al (2011) White to brown fat phenotypic switch induced by genetic and environmental activation of a hypothalamic-adipocyte axis. Cell Metab 14:324-338

30. Ohno H, Shinoda K, Spiegelman BM, Kajimura S (2012) PPARgamma agonists induce a white-to-brown fat conversion through stabilization of PRDM16 protein. Cell Metab 15:395-404

31. Bostrom P, Wu J, Jedrychowski MP et al (2011) A PGC1-alphadependent myokine that drives brown-fat-like development of white fat and thermogenesis. Nature 481:463-468

32. Bordicchia M, Liu D, Amri EZ et al (2012) Cardiac natriuretic peptides act via p38 MAPK to induce the brown fat thermogenic program in mouse and human adipocytes. J Clin Invest 122:10221036

33. Wang QA, Tao C, Gupta RK, Scherer PE (2013) Tracking adipogenesis during white adipose tissue development, expansion and regeneration. Nat Med 19:1338-1344

34. Dempersmier J, Sambeat A, Gulyaeva O et al (2015) Coldinducible Zfp516 activates UCP1 transcription to promote browning of white fat and development of brown fat. Mol Cell 57:235246

35. McDonald ME, Li C, Bian H et al (2015) Myocardin-related transcription factor a regulates conversion of progenitors to beige adipocytes. Cell 160:105-118

36. Cohen P, Levy JD, Zhang Y et al (2014) Ablation of PRDM16 and beige adipose causes metabolic dysfunction and a subcutaneous to visceral fat switch. Cell 156:304-316

37. She P, Burgess SC, Shiota M et al (2003) Mechanisms by which liver-specific PEPCK knockout mice preserve euglycemia during starvation. Diabetes 52:1649-1654

38. Kurlawalla-Martinez C, Stiles B, Wang Y et al (2005) Insulin hypersensitivity and resistance to streptozotocin-induced diabetes in mice lacking PTEN in adipose tissue. Mol Cell Biol 25:2498-2510
39. Kumar A, Lawrence JC Jr, Jung DY et al (2010) Fat cell-specific ablation of rictor in mice impairs insulin-regulated fat cell and whole-body glucose and lipid metabolism. Diabetes 59:1397-1406

40. Shepherd PR, Kahn BB (1999) Glucose transporters and insulin action-implications for insulin resistance and diabetes mellitus. N Engl J Med 341:248-257

41. Yore MM, Syed I, Moraes-Vieira PM et al (2014) Discovery of a class of endogenous mammalian lipids with anti-diabetic and antiinflammatory effects. Cell 159:318-332

42. Lee DV, Li D, Yan Q et al (2014) Fibroblast growth factor 21 improves insulin sensitivity and synergizes with insulin in human adipose stem cell-derived (hASC) adipocytes. PLoS One 9: e111767

43. Riserus U, Willett WC, Hu FB (2009) Dietary fats and prevention of type 2 diabetes. Prog Lipid Res 48:44-51

44. Rhee EP, Cheng S, Larson MG et al (2011) Lipid profiling identifies a triacylglycerol signature of insulin resistance and improves diabetes prediction in humans. J Clin Invest 121:1402-1411

45. Burhans MS, Flowers MT, Harrington KR et al (2015) Hepatic oleate regulates adipose tissue lipogenesis and fatty acid oxidation. J Lipid Res 56:304-318

46. Muir K, Hazim A, He Y et al (2013) Proteomic and lipidomic signatures of lipid metabolism in NASH-associated hepatocellular carcinoma. Cancer Res 73:4722-4731

47. Summers SA (2011) Sphingolipids and insulin resistance: the five Ws. Curr Opin Lipidol 21:128-135

48. Cao H, Gerhold K, Mayers JR et al (2008) Identification of a lipokine, a lipid hormone linking adipose tissue to systemic metabolism. Cell 134:933-944

49. Perry RJ, Camporez J-PG, Kursawe R et al (2015) Hepatic acetyl CoA links adipose tissue inflammation to hepatic insulin resistance and type 2 diabetes. Cell 160:745-758

50. Consoli A, Nurjhan N, Capani F, Gerich J (1989) Predominant role of gluconeogenesis in increased hepatic glucose production in NIDDM. Diabetes 38:550-557 